\title{
Análise da estacionariedade do sinal de eletromiografia de superfície nas fases do exercício isocinético de extensão do joelho
}

\author{
Fabiano Peruzzo Schwartz*, Francisco Assis de Oliveira Nascimento, Martim Bottaro, \\ Rodrigo Souza Celes
}

Resumo A dinamometria isocinética (DI) e a eletromiografia de superfície (EMG-S) são duas técnicas bastante utilizadas no estudo da força muscular. Apesar do reconhecido potencial de ambas, sua eficácia está condicionada ao bom entendimento de suas limitações. No caso da DI é importante considerar o movimento apenas durante a região de carga, ou seja, quando o dinamômetro impõe resistência à articulação em exercício de forma a manter constante a sua velocidade angular, independentemente da força aplicada pelo indivíduo submetido ao teste. Contudo, no início da região de carga ocorrem significativas oscilações da velocidade angular quando o membro em aceleração é conduzido ao estado de velocidade constante. Essa região de oscilação afeta consideravelmente a estimativa das variáveis biomecânicas de torque, velocidade e posição angular. Quanto aos sinais de EMG-S, sabe-se que em condições isométricas podem ser considerados estacionários no sentido amplo, suposição que não se aplica ao caso dinâmico em que o conteúdo de frequência do sinal muda ao longo do tempo. Técnicas utilizadas para minimizar os efeitos não-estacionários consistem em limitar a análise a um intervalo cíclico em que as mudanças mecânicas do músculo se repetem periodicamente. Nesse contexto, o presente trabalho avaliou as condições de estacionariedade do sinal de EMG-S na região de carga do exercício isocinético, visando estabelecer um intervalo dinâmico cíclico adequado ao estudo desses sinais quando adquiridos durante testes isocinéticos de extensão do joelho. Os resultados encontrados sugerem que a análise da EMG-S seja efetuada somente no trecho da região de carga em que a velocidade angular é estável.

Palavras-chave Dinamometria isocinética, Eletromiografia de superfície, Estacionariedade de sinais, Região de carga.

\section{Stationarity analysis of surface electromyographic signals on the phases of the isokinetic knee extension exercise}

\footnotetext{
Abstract Isokinetic dynamometry (ID) and surface electromyography (SEMG) are two widely used techniques in the study of muscular strength. However, the effectiveness of both is conditioned to the good understanding of their particularities and limitations. For example, resulting data from ID tests must be considered valid only on the load range phase which is the part of the exercise where the dynamometer imposes resistance against the exercising limb in order to keep it at constant angular velocity. Data from acceleration and deceleration phases are often included in analyses and may result in spurious conclusions. Moreover, significant fluctuations of angular velocity in the beginning of the load range can considerably affect the estimate of biomechanical variables such as torque, speed and angular position. Regarding SEMG signals, they can be considered stationary in the broad sense when acquired under isometric conditions. This assumption cannot be directly applied to the case of dynamic contractions since the frequency content of the signal changes over time in this situation. Some techniques are used to minimize non-stationary effects by setting out a range in which the mechanical changes of muscles are cyclic, i.e. they are repeated periodically. Based on the features of ID and SEMG, this study aimed to find out a dynamic range with suitable stationarity conditions for the study of SEMG signals during cyclical isokinetic knee extension tests. The results showed that SEMG analyses should consider only the portion of the load range where angular velocity is stable, i.e. with no significant fluctuations.
}

Keywords Isokinetic dynamometry, Surface electromyography, Stationarity of signals, Load range. 


\section{Extended Abstract}

\section{Introduction}

When the surface myoelectric signal is recorded during a constant-force, isometric contraction, it may be considered as a realization of a wide-sense stationary stochastic process with Gaussian amplitude distribution and zero mean (Basmajian and De Luca, 1985). However, under dynamic conditions, the assumption of stationarity does not hold because the frequency content of the signal continuously changes over time (Bonato et al., 2001) as a consequence of variations in muscle force (Bilodeau et al., 1990; Broman et al., 1985), changes in muscle length (Inbar et al., 1987), and the relative displacement of electrodes with respect to the underlying muscle fibers (Farina et al., 2004). Therefore, appropriate methods of digital signal processing must be used for non-stationary signals study. One useful technique consists of setting out the analysis to a dynamic activity that is mechanically reproducible (Bonato et al., 2001). In the case of isokinetic exercises, it is reasonable to assume that load range is the best portion of the available range of motion for signal analyses since the limb movement occurs at constant angular velocity. However, nothing was found in the literature about the frequency content of SEMG signals regarding the isokinetic exercise phases.

For this reason, the present study evaluated the stationarity conditions of SEMG signals on the load range phase that was divided in two segments (Figure 1): velocity overshoot (VO), which is the portion where the limb angular velocity strongly oscillates after acceleration phase; isokinetic load range (ILR), which is the portion where the limb angular velocity is nearly constant. The main goal was to find the most suitable range for the study of SEMG signals during isokinetic knee extension tests.

\section{Materials and Methods}

Sixteen normal healthy adult males (age $26.8 \pm 4.7$ years) of height $1.76 \pm 0.05 \mathrm{~m}$ and body mass $79.2 \pm 9.4 \mathrm{~kg}$ with no history of orthopedic disease voluntarily participated in this study. They were asked to perform three sets of ten maximal concentric repetitions of dominant knee extension (at $60 \%$ and $180 \%$ s) on a calibrated Biodex System 3 Pro (Biodex Corp., Shirley, NY, USA) isokinetic dynamometer. The SEMG signals were acquired from the vastus lateralis muscle with a linear adhesive array consisting of eight electrodes (5 $\mathrm{mm}$ inter-electrode distance), amplified in the single differential mode by a multichannel amplifier (EMG 16, LISiN - OT Bioelettronica Snc, Torino, Italy), bandpass filtered $(-3 \mathrm{~dB}$ bandwidth $=10-500 \mathrm{~Hz}$ ) with a $4^{\text {th }}$ order Bessel filter, sampled at 2048 samples/s, and converted to digital data by a 12 bit A/D converter board. Computational tools and algorithms (Schwartz et al., 2011) were used to extract the segments of SEMG signal matching $V O$ and ILR phases (at $60 \%$ s and $180 \%$ s) from each of the ten repetitions of the three performed isokinetic sets.

Firstly, the KPSS stationarity test (Kwiatkowiski et al., 1992) was applied to the segments of VO and ILR with significance level of 0.05. Whenever the null hypothesis is rejected by KPSS ( $H_{0}$-non-stationary signal) the test returns the value 1 (one). On the other hand, when the signal is stationary, the returned value is 0 (zero). Secondly, the average of the returned values was estimated among all the subjects for VO and ILR, for each repetition of each exercise set, according to the scheme of Table 2. Finally, the mean ( $\pm S D$ ) of the previous estimates was calculated for each isokinetic set, by using the Equation 7, and it was used as an indicator of stationarity (Figure 2): the closer to zero, the bigger the level of the signal stationarity; the closer to one, the lower the level of the signal stationarity. Statistic comparisons between the phases of VO and ILR, as well as among the estimates of the three exercise sets, used $t$-Student test (for two groups with dependent samples and normal distribution), Wilcoxon-Signed-Rank test (for those with non-normal distribution), and Friedman test with posthoc Wilcoxon-Signed-Rank (for three groups of non-normal distribution).

\section{Results and Discussion}

The results indicated a high degree of non-stationarity in $\mathrm{VO}$ when compared with ILR. Table 3 shows that VO averages are within the range of non-stationarity $(0.51]$ whereas ILR averages are within the stationarity range [0 0.5). This fact is consistent with Table 5, where the VO estimates are statistically higher than those of the ILR phase, for the three isokinetic sets and for both angular velocities. The ratio between the averages of VO and ILR approximately varies from 8 to 20 (3 to 5) at $60 \%$ $(180 \% \mathrm{~s})$, which means that non-stationary factors have the most significant impact on VO. Therefore, VO is not an appropriate range for the study of SEMG signals. On the other hand, the estimated values on ILR are very close to zero (especially at $60 \%$ s) which means there is a cycle-stationary behavior. Hence, ILR can be considered a suitable range for SEMG analysis.

Table 3 also shows that averages at $180 \%$ s are higher than those at $60 \%$, suggesting that the higher the angular velocity, the higher the non-stationarity of the SEMG signal. This hypothesis is confirmed by Table 6 which shows that non-stationary effects can be up to 10 (1.73) times higher for ILR (VO) when $180 \%$ is compared with $60 \%$ s. As the inertia of the exercising limb at $180 \%$ is greater than at $60 \%$, the dynamometer needs extra effort to adjust angular velocity to a constant level, producing additional motion artifacts.

When the same isokinetic phase was compared among the exercise sets of a same angular velocity, as shown in Table 4, there was an only significant difference between the sets 1 and 3 for ILR $\left(180^{\circ} / \mathrm{s}, \mathrm{S1}<\mathrm{S3}\right)$. The higher level of non-stationarity in set 3 , at $180 \%$, is due to the more pronounced state of fatigue. This difference is not seen at $60 \%$ secause the load range is longer and the fatigue state usually starts in the first set. The results showed that under dynamic contractions the disruptive effects on the SEMG signal are cyclical and almost invariant, occurring intensively only in VO. Therefore, SEMG signals can be considered cycle-stationary in ILR.

\section{Conclusion}

High levels of non-stationarity were found in VO against significant levels of stationarity in ILR. This fact strengthens the hypothesis that the analysis of SEMG signals is only advisable in the range corresponding to ILR. 


\section{Introdução}

Quando um sinal eletromiográfico é adquirido durante uma contração isométrica constante, ele pode ser considerado como a realização de um processo estocástico estacionário no sentido amplo (widesense stationary) com distribuição Gaussiana da amplitude e média zero (Basmajian e De Luca, 1985). Contudo, sob condições dinâmicas a suposição de estacionariedade não se aplica visto que o conteúdo de frequência do sinal muda ao longo do tempo. Não-estacionariedades do sinal de EMG-S podem ser classificadas como lentas ou rápidas (Bonato et al., 2001). As lentas são, normalmente, devido ao acúmulo de metabólitos que causam as manifestações elétricas da fadiga muscular. Esses metabólitos afetam o pH dos fluidos intersticiais do músculo, o que implica na redução da velocidade de condução do potencial de ação. As rápidas são, em geral, relacionadas aos fatores biomecânicos (Bonato et al., 2001). O ângulo das articulações, a posição dos membros, o comprimento dos músculos e a força muscular variam continuamente (Broman et al., 1985; Inbar et al., 1987), enquanto vários músculos trabalham em conjunto para realizar certo movimento. Isso pode resultar em rápidas mudanças no recrutamento/desrecrutamento de unidades motoras, o que poderia introduzir nãoestacionariedades no sinal de EMG-S. Além disso, os músculos em contração dinâmica frequentemente mudam sua posição em relação aos eletrodos de aquisição (Farina et al., 2004).

Um método utilizado para minimizar os efeitos não-estacionários e melhorar a sensibilidade de estimativas tempo-frequência do sinal de EMG-S é a limitação da análise a uma atividade dinâmica mecanicamente reproduzível. Nesse caso, somente são consideradas as contrações dinâmicas cíclicas, que resultam em mudanças mecânicas do músculo que se repetem periodicamente. Assume-se que, nessas condições, existe uma faixa da atividade muscular em que as variações da força, do comprimento do músculo e da velocidade de encurtamento/alongamento das fibras musculares são praticamente idênticas (Bonato et al., 2001).

Exercícios de dinamometria isocinética são caracterizados por repetições cíclicas. Por essa razão, o estudo da EMG-S associado a esses exercícios deve ser focado sobre uma faixa do movimento em que os efeitos não-estacionários sejam amenizados. A definição de critérios para a identificação dessa faixa representa um desafio inexplorado quando se combinam as técnicas de DI e de EMG-S. Esforços nesse sentido podem contribuir para se restringirem, em parte, as causas de não-estacionariedades rápidas.
Ao se considerar que em determinada porção do ciclo os fatores não-estacionários são invariantes, pode-se supor que o surgimento de novos níveis de nãoestacionariedades no sinal de EMG-S tenha origem em causas de natureza lenta, ou seja, em fenômenos como a fadiga muscular, por exemplo. Significa dizer que durante um número determinado de ciclos as características permanecem aproximadamente estáveis (ao que se denomina ciclo-estacionariedade) até que novos fenômenos alterem essa condição.

Nesse contexto, o objetivo deste trabalho consistiu em investigar a região de carga do exercício isocinético (Figura 1) como intervalo dinâmico cíclico adequado ao estudo dos sinais de EMG-S em testes isocinéticos de extensão do joelho. A estacionariedade dos sinais mioelétricos foi avaliada por meio do teste KPSS aplicado em dois segmentos da região de carga: o artefato de oscilação da velocidade (velocity overshoot - VO) e o segmento de velocidade constante (isokinetic load range - ILR). Foram testadas: 1) a necessidade de descarte da informação do sinal mioelétrico contido em VO; e 2) a viabilidade do segmento ILR como a faixa mais apropriada ao estudo da EMG-S na dinamometria isocinética. A motivação adveio do estudo de Schwartz et al. (2010) que quantificou o impacto de VO sobre os indicadores biomecânicos, sugerindo o descarte da informação contida em VO quando da análise desses indicadores.

\section{Fases do exercício isocinético}

O dinamômetro isocinético é um dispositivo que controla a velocidade do exercício. Quanto mais força é exercida sobre a alavanca do dinamômetro, mais energia do membro em movimento é absorvida pelo mecanismo de controle e retornada como resistência adicional ao movimento (Brown et al., 1995a;b). Dessa forma, o movimento ocorre a uma velocidade constante pré-determinada durante a faixa de movimento denominada região de carga (Brown, 2000) (Figura 1). No exercício isocinético, o membro acelera livremente (acceleration - ACC) até a velocidade pré-determinada e desacelera (deceleration - DEC) no final da faixa até o ponto de extensão máxima do exercício (Sapega et al., 1982). Essas duas etapas são realizadas sem o benefício da resistência externa imposta pelo dinamômetro e, consequentemente, não devem ser consideradas durante a interpretação dos testes isocinéticos (Brown, 2000). Na região de carga, a velocidade é constante apenas no segmento onde a carga imposta se iguala à força muscular exercida (ILR) (Schwartz et al., 2010). Há um trecho em que a velocidade oscila, quando o dinamômetro ainda está se ajustando à força aplicada, denominado artefato de oscilação da velocidade (VO) (Figura 1). 


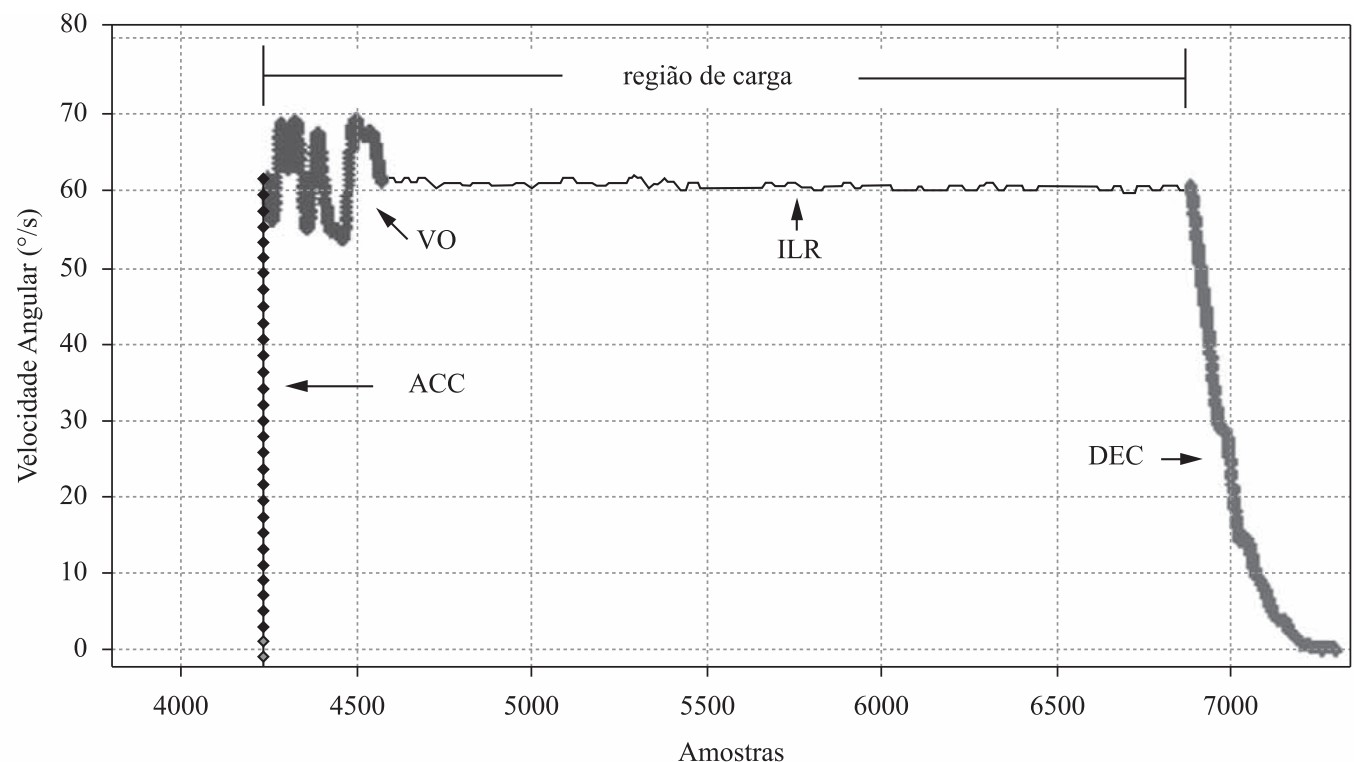

Figura 1. Sinal da velocidade angular com a representação das fases do exercício isocinético de extensão do joelho a $60^{\circ} / \mathrm{s}$ : aceleração (acceleration - ACC), artefato de oscilação da velocidade (velocity overshoot - VO), velocidade constante na região de carga (isokinetic load range - ILR) e desaceleração (deceleration - DEC).

Figure 1. Angular velocity signal with the respective phases of the isokinetic knee extension exercise at $60 \%$ s: acceleration (ACC), velocity overshoot (VO), isokinetic load range (ILR) and deceleration (DEC).

\section{Estacionariedade e integração}

Um processo estacionário no sentido estrito (strictsense stationary) é um processo estocástico cuja distribuição de probabilidade não varia ao longo do tempo (Komo, 1987). Características básicas como a média $\left(E\left(Y_{t}\right)\right)$ e a variância $\left(\operatorname{Var}\left(Y_{t}\right)\right)$ permanecem constante ao longo do tempo. Séries temporais estacionárias são mais fáceis de analisar e prever. Por essa razão, séries originalmente não-estacionárias são frequentemente transformadas em séries estacionárias para futuras análises. Processos não-estacionários apresentam uma tendência que é um componente sistemático, linear ou não, que muda ao longo do tempo e não se repete, pelo menos durante a faixa de tempo em que ocorre a captura dos dados. Há dois tipos de tendência: determinística e estocástica. Séries temporais com tendência determinística têm variância constante e média variante, enquanto que as séries com tendência estocástica exibem variância não constante. Alguns processos podem conter ambas as tendências.

Um processo não-estacionário com tendência determinística é transformado em um processo estacionário através de sua regressão no tempo. Já para um processo não-estacionário com tendências estocásticas, o método mais comum para a remoção dessas tendências é a diferenciação. A diferenciação de uma série temporal $Y_{t}$ no tempo discreto - ou a sua derivada discreta (Smith, 1997) - é uma nova série $d i f_{t}$ na qual os seus valores são as diferenças entre valores consecutivos de $Y_{t}$. A diferenciação de ordem $n$ é descrita por:

$$
\operatorname{dif}^{(n)}{ }_{t}=\operatorname{dif}^{(n-1)}{ }_{t}-\operatorname{dif}^{(n-1)}{ }_{t-1}
$$

Algumas séries precisam ser diferenciadas várias vezes até atingirem a estacionariedade. Deste fato vem a definição de integração: uma série temporal é dita integrada de ordem $n$ (ou $I(n)$ ) quando ela se torna estacionária depois de ser diferenciada $n$ vezes. Uma série $I(n)$ é dita ter $n$ raízes unitárias.

\section{Teste de estacionariedade KPSS}

O KPSS foi originalmente desenvolvido para o estudo de séries temporais relacionadas às ciências econômicas (Kwiatkowiski et al., 1992). Contudo, seu uso tem se mostrado adequado a sinais biofísicos como os oriundos de eletroencefalografia (Maganioti et al., 2010).

Testes de estacionariedade verificam a hipótese nula de que uma série $Y_{t}$ é $I(0)$. Considere que $Y_{t}$, $t=1, \ldots, n$, é a série temporal observada, para a qual se deseja testar a estacionariedade, e que $\bar{Y}_{n}$ é a média das amostras de $Y_{t}$. Considere, também, o processo de soma parcial das derivações a partir da média estimada:

$$
S_{n}(u)=\sum_{t=1}^{u}\left(Y_{t}-\bar{Y}_{n}\right)
$$


A hipótese nula da estacionariedade é formulada como $H_{0}: E\left[S_{n}(u)\right]=0$ para todo $u=1, \ldots, n$. Se o processo é não-estacionário, contudo, pode-se esperar que $E\left[S_{n}(u)\right]$ seja diferente de zero para algum $u$. Em termos do processo de soma parcial $S_{n}(u)$, Kwiatkowiski et al. (1992) propuseram o seguinte teste estatístico KPSS para a estacionariedade:

$$
T_{n}=\frac{1}{n^{2} S_{n}^{2}} \sum_{u=1}^{n}\left[S_{n}(u)\right]^{2}
$$

onde

$$
s_{n}^{2}=m_{n}^{-1} \sum_{t, j=1}^{m_{n}} \hat{\gamma}_{t-j}
$$

$\hat{\gamma}_{j}=(n-j)^{-1} \sum_{t=1}^{n-j}\left(Y_{t}-\bar{Y}_{n}\right)\left(Y_{t+j}-\bar{Y}_{n}\right)$ para $0 \leq j<n$

e $m_{n}$ é uma largura de banda que satisfaz $m_{n} \rightarrow \infty$ e $m_{n} / n \rightarrow 0$. Aqui $s_{n}^{2}$ é um estimador não-paramétrico para o limite de $n \operatorname{Var}\left(\bar{Y}_{n}\right)$, desde que ele exista. Sob certas condições brandas, Kwiatkowiski et al. (1992) mostraram que $T_{n}$ convergiu em distribuição para:

$$
K P S S \rightarrow \int_{0}^{1} V(t)^{2} d t
$$

onde $V(t)$ é uma ponte de Brownian padrão (Papoulis e Pillai, 2002). O teste pode então ser conduzido pela comparação de $T_{n}$ com os valores críticos da cauda superior da integral na Equação 6, os quais podem ser aproximados pela simulação da ponte de Brownian $V(t)$, como exemplifica a Tabela 1. Especificamente, se $T_{n}$ é maior que o valor crítico desejado da cauda superior, então a estacionariedade deve ser rejeitada.

Estudos têm mostrado (Hobijn et al., 2004; Kwiatkowiski et al., 1992; Maganioti et al., 2010) que o teste KPSS é o mais poderoso teste de estacionariedade para séries temporais. Detalhes adicionais do teste KPSS são descritos no trabalho de Zivot e Wang (2002).

\section{Materiais e Métodos}

Um protocolo para a execução de exercício resistido isocinético de intensidade máxima, baseado em contrações dinâmicas de extensão do joelho nas velocidades de $60 \%$ s e $180^{\circ} / \mathrm{s}$, foi configurado na arquitetura de instrumentação proposta por Schwartz et al. (2011), seguindo práticas reconhecidas do teste físico isocinético (Brown, 2000) e da aquisição eletromiográfica de sinais (De Luca, 1997). Participaram do experimento 16 (dezesseis) sujeitos saudáveis e do sexo masculino, com idade de $26,8 \pm 4,7$ anos, altura de $1,76 \pm 0,05 \mathrm{~m}$ e massa corporal de 79,2 $\pm 9,4 \mathrm{~kg}$, sem histórico de doenças ortopédicas. Eles leram e assinaram voluntariamente um termo de consentimento antes de participar do experimento, o qual foi aprovado pelo Comitê de
Tabela 1. Quantis da distribuição estatística de KPSS.

Table 1. Quantiles of the distribution of the KPSS statistic.

Quantis da cauda direita

\begin{tabular}{llcc}
\hline Distribuição & $\mathbf{0 , 9 0}$ & $\mathbf{0 , 9 5}$ & $\mathbf{0 , 9 9}$ \\
\hline $\int_{0}^{1} V(t)^{2} d t$ & 0,349 & 0,446 & 0,762
\end{tabular}

Nota: Resultados da simulação da ponte de Brownian $V(t)$ efetuada por Zivot e Wang (2002).

Note: Results of the simulation of the Brownian's bridge $V(t)$ performed by Zivot and Wang (2002).

Ética da Faculdade de Saúde da Universidade de Brasília (UnB).

Um dinamômetro isocinético (Biodex Corporation, modelo Biodex System 3 Pro) foi calibrado e preparado para a realização de exercícios controlados da articulação do joelho no modo isocinético concêntrico (Brown, 2000). O software de controle usado para o dinamômetro foi o System 3 (Biodex Medical Systems, v. 3.40 de 17/05/2006). A articulação do joelho foi passivamente movida até a posição $0^{\circ}$ de extensão, o que corresponde à máxima extensão possível. Depois, o joelho foi flexionado cerca de 5 a $10^{\circ}$ para uma posição confortável, registrada no software de controle do dinamômetro como o ponto de extensão máxima para o experimento (parada mecânica da extensão). Então, o ponto de flexão máxima (parada mecânica da flexão) foi definido de modo a garantir uma faixa de movimento de $85^{\circ}$. A análise de compensação da gravidade foi realizada pelo software de controle do Biodex System 3 Pro. A interface DB-15 do dinamômetro foi conectada a um adaptador (construído pelos autores) com três saídas $\mathrm{BNC}$ (uma para cada sinal biomecânico) ligadas a um conversor A/D (National Instruments, modelo BNC-2120) que digitalizou os sinais biomecânicos a 2.048 amostras/s e resolução de 12 bits, com auxílio do software de controle LabVIEW ${ }^{\circledR}$ v. 5.1 (National Instruments). Esse procedimento foi estabelecido para se adequar a resolução temporal dos sinais biomecânicos à dos sinais de EMG-S - condição necessária para a análise conjunta - visto que a conversão $\mathrm{A} / \mathrm{D}$ interna do dinamômetro isocinético ocorre a apenas 100 amostras/s.

Os sinais de EMG-S foram adquiridos durante as contrações do músculo vastus lateralis por meio de um arranjo linear flexível de oito eletrodos (Ottino Bioelettronica com $5 \mathrm{~mm}$ de distância entre os eletrodos), amplificados (com ganho 2.000) por um eletromiógrafo multicanal (LISiN-OT Bioelettronica Snc, modelo EMG-16) configurado para digitalizar os sinais à taxa de 2.048 amostras/s (resolução de 12 bits) e filtrados por um filtro de Bessel passa-faixa de $4^{\mathrm{a}}$ ordem e largura de banda de $10-500 \mathrm{~Hz}$ a $-3 \mathrm{~dB}$. O software de controle utilizado com o EMG-16 foi o 
Emgacq v. 1.0 (Centro do Bioingegneria, Politecnico di Torino). Precedendo a colocação do arranjo flexível, o músculo vastus lateralis foi avaliado em uma contração isométrica, com a alavanca do dinamômetro posicionada em $60^{\circ}$, utilizando-se um arranjo linear seco de 16 eletrodos (Ottino Bioelettronica com barras retangulares de $\mathrm{Ag}-\mathrm{AgCl}$ de $5 \mathrm{~mm}$ de comprimento e $1 \mathrm{~mm}$ de largura, separadas por $5 \mathrm{~mm}$ de distância). $\mathrm{O}$ arranjo seco foi movido sobre o músculo para a localização da zona de inervação (Farina et al., 2004; Masuda et al., 1985) e posterior posicionamento do arranjo de 8 eletrodos entre a zona de inervação e a região dos tendões (De Luca, 1997), paralelamente à direção das fibras musculares. Em particular, a zona de inervação corresponde ao ponto em que ocorre a inversão da propagação do potencial de ação da unidade motora (Farina et al., 2004), o que pode ser identificado visualmente com o auxílio do software Emgacq.

Um sensor de posição composto por uma chave magnética (fixada no corpo do dinamômetro) e um ímã (fixado na haste de movimento) registrou o tempo exato de cada extensão do joelho, enviando essa informação simultaneamente ao eletromiógrafo e ao conversor A/D na forma de um trem de pulsos.

Montados os equipamentos, cada sujeito realizou 3 (três) séries de 10 (dez) repetições concêntricas máximas de extensão a $60 \%$ s, com descanso de 1 minuto entre as séries. Após 20 (vinte) minutos as 3 (três) séries foram repetidas a $180^{\circ} / \mathrm{s}$, com o mesmo descanso entre as séries. Um comando verbal consistente e moderado (sem gritos) foi realizado durante a execução de cada série - com a finalidade de obter o máximo rendimento de cada sujeito - e nenhum retorno visual sobre o torque produzido em cada repetição ficou disponível aos participantes (McNair et al.,1996).

Com o auxílio da interface computacional (Schwartz et al., 2008) construída para a instrumentação utilizada e para os algoritmos de análise de sinais propostos por Schwartz et al. (2011), os sinais de EMG-S e biomecânicos foram sincronizados - com base no trem de pulsos - e os segmentos de sinal de EMG-S, relativos às etapas VO e ILR, foram extraídos das 10 (dez) repetições em todas as 3 (três) séries, para ambas as velocidades angulares $\left(60^{\circ} / \mathrm{s} \mathrm{e} 180^{\circ} / \mathrm{s}\right)$ e para todos os sujeitos. O critério de escolha do canal de EMG-S se deu pela verificação da relação sinalruído (signal to noise ratio) com o método do ruído do canal ocioso (idle channel noise) (Schwartz et al., 2011). Nessa técnica, o sinal de ruído foi captado com o arranjo de eletrodos já fixado sobre o músculo, entretanto, com o sujeito em estado relaxado (sem contração muscular voluntária).
A estacionariedade do sinal de EMG-S foi verificada em cada segmento extraído, utilizando-se o teste KPSS. O algoritmo KPSS realiza uma regressão para encontrar os mínimos quadrados lineares que melhor ajustam os sinais de EMG-S ao modelo da hipótese nula (Kwiatkowiski et al., 1992). Nesse processo não é necessário nenhum janelamento ou normalização do sinal de EMG-S. Sempre que a aplicação do KPSS rejeitou a hipótese nula $\left(H_{0}\right)$, por convenção atribuiu-se o valor 1 (um) ao resultado do teste. Quando houve aceitação de $H_{0}$, o valor atribuído ao resultado foi 0 (zero), significando que a série temporal é estacionária. O nível de significância adotado foi de 0,05 .

Para cada repetição de uma série isocinética, e para cada sujeito, determinou-se a média dos resultados retornados pelo teste KPSS nas etapas VO e ILR, conforme o esquema da Tabela 2.

Então foi calculada, para cada etapa, a média ( \pm desvio padrão) da série isocinética, a qual corresponde à média ( \pm desvio padrão) das médias em cada repetição, como detalha a Equação 7.

$$
M_{\text {VOsérie }}=\frac{1}{10} \sum_{i=1}^{10} M_{\text {VOi }}, M_{\text {ILRsérie }}=\frac{1}{10} \sum_{i=1}^{10} M_{\text {ILRi }}
$$

Neste estudo se propõe considerar a média da série isocinética como um indicador do nível de estacionariedade do sinal de EMG-S dentro da série (Figura 2): quanto mais próxima de 0 (zero), maior o nível de estacionariedade; quanto mais próxima de 1 (um), menor.

Comparações estatísticas foram realizadas entre as médias de cada etapa (VO e ILR) dentro de uma série, entre as três séries e entre as velocidades angulares, com nível de significância de 0,05 (cauda dupla) e $95 \%$ de intervalo de confiança. Utilizou-se o teste de normalidade de Shapiro-Wilk, confiável para números pequenos de amostra (em torno de 10)

Tabela 2. Método para estimar a média dos valores retornados pelo teste KPSS aplicado sobre VO e ILR em cada repetição da série isocinética $s$.

Table 2. Method for estimating the average of the values returned by the KPSS test applied on VO and ILR for each iteration of the isokinetic set $s$.

\begin{tabular}{|c|c|c|c|c|c|c|c|}
\hline \multirow{3}{*}{ Sujeito } & \multicolumn{7}{|c|}{ Série } \\
\hline & \multicolumn{2}{|c|}{ Rep. 1} & \multicolumn{2}{|c|}{ Rep. 2} & \multirow[t]{2}{*}{.} & \multicolumn{2}{|c|}{ Rep. 10} \\
\hline & VO & ILR & VO & ILR & & VO & ILR \\
\hline S01 & 1 & 0 & 0 & 0 &. & 1 & 0 \\
\hline $\mathrm{S} 02$ & 0 & 0 & 1 & 0 &. & 1 & 0 \\
\hline . & . & . & . & . & & . & . \\
\hline . & . & . & . & . & & . & . \\
\hline . & . & . & . & . & & . & . \\
\hline S16 & 1 & 0 & 1 & 0 & . . & 0 & 0 \\
\hline Média & $\mathrm{M}_{\mathrm{VO} 1}$ & $\mathrm{M}_{\mathrm{ILR} 1}$ & $\mathrm{M}_{\mathrm{VO} 2}$ & $\mathrm{M}_{\mathrm{ILR} 2}$ &. & $\mathrm{M}_{\mathrm{VO} 10}$ & $\mathrm{M}_{\mathrm{ILR} 10}$ \\
\hline
\end{tabular}


(De Sá, 2007). Na comparação das médias entre duas amostras (p.e., dos níveis de estacionariedade de VO e ILR na mesma série) foi usado o teste $t$-Student para amostras dependentes - distribuição normal - e o teste não-paramétrico Wilcoxon-Signed-Rank - distribuição não-normal. Na comparação entre as médias de três amostras (p.e., dos níveis de estacionariedade de VO em cada uma das três séries isocinéticas) foi usado o teste de Friedman com Wilcoxon Signed-Rank (post hoc) - não-normal.

\section{Resultados}

A Tabela 3 mostra, para cada célula de resultado, a média de respostas do teste KPSS entre os 16 (dezesseis) sujeitos (conforme sugerido na Tabela 2), consolidando-as na média de cada etapa (VO e ILR) dentro da série isocinética, conforme a Equação 7 (p.e., a média dos 16 sujeitos em $\mathrm{VO}$, a $60 \%$ s, na repetição 1 da série 1 é igual a 0,75$)$. A Tabela 4 compara o nível de estacionariedade (sinal de EMG-S) entre as séries, respectivamente, para cada etapa (VO e ILR), considerando a mesma velocidade angular. A Tabela 5 compara as etapas VO e ILR dentro de cada série isocinética quanto aos níveis de estacionariedade. A Tabela 6 compara o nível de estacionariedade entre as velocidades $60 \%$ s e $180 \%$ s, para cada uma das etapas VO e ILR, dentro de cada série isocinética.

\section{Discussão}

Os resultados decorrentes da aplicação do método proposto para o estudo da estacionariedade dos sinais de EMG-S no exercício isocinético apontam para um grau elevado de não-estacionariedade na etapa VO, quando comparado com a etapa ILR.

Em primeira análise, pode-se observar na Tabela 3 que as médias referentes à etapa VO são superiores a 0,5 tanto em $60 \%$ s quanto em $180 \%$ s, localizando-se no intervalo de não-estacionariedade $(0,5-1]$. Já para a etapa ILR, as médias estão dentro do intervalo de estacionariedade [0-0,5), caracterizando maior estabilidade dos sinais nessa faixa. Esses resultados são ratificados pela Tabela 5 em que os testes estatísticos confirmaram a diferença entre as etapas VO e ILR, nas 3 (três) séries isocinéticas e em ambas as velocidades angulares, com a média em VO sempre maior que em ILR. A relação entre as médias de VO e ILR varia, aproximadamente, de 8 a 20 (de 3 a 5 ) a $60 \%$ (a $180^{\circ}$ s). Isso indica que os fatores não-estacionários são significativamente mais impactantes em VO do que em ILR, revelando a inadequação da faixa VO para o

\section{$\rightarrow$ Média do teste KPSS}

0,5

\section{Estacionário}

Indefinido

Não-estacionário

Figura 2. Indicador do nível de estacionariedade com base na média do teste KPSS.

Figure 2. Indicator of the level of stationarity based on the average of the KPSS test.

Tabela 3. Nível de estacionariedade em cada etapa (VO e ILR) e para cada série isocinética, nas velocidades de $60 \%$ s e $180 \%$ s. Table 3. Stationarity level at each segment (VO and ILR) for each isokinetic set and each angular velocity (60\% and $180 \%$ s).

\begin{tabular}{|c|c|c|c|c|c|c|c|c|c|c|c|c|}
\hline \multirow{3}{*}{ Repetição } & \multicolumn{6}{|c|}{$60 \% / s$} & \multicolumn{6}{|c|}{$180 \% / \mathrm{s}$} \\
\hline & \multicolumn{2}{|c|}{ Série 1} & \multicolumn{2}{|c|}{ Série 2} & \multicolumn{2}{|c|}{ Série 3} & \multicolumn{2}{|c|}{ Série 1} & \multicolumn{2}{|c|}{ Série 2} & \multicolumn{2}{|c|}{ Série 3} \\
\hline & VO & ILR & VO & ILR & VO & ILR & VO & ILR & VO & ILR & VO & ILR \\
\hline 1 & 0,75 & 0,19 & 0,94 & 0,25 & 0,94 & 0,13 & 0,94 & 0,31 & 0,94 & 0,19 & 1,00 & 0,38 \\
\hline 2 & 0,69 & 0,06 & 0,44 & 0,00 & 0,69 & 0,00 & 1,00 & 0,19 & 0,94 & 0,31 & 1,00 & 0,25 \\
\hline 3 & 0,50 & 0,13 & 0,69 & 0,00 & 0,56 & 0,00 & 1,00 & 0,25 & 1,00 & 0,25 & 1,00 & 0,31 \\
\hline 4 & 0,69 & 0,00 & 0,56 & 0,06 & 0,63 & 0,06 & 1,00 & 0,19 & 1,00 & 0,25 & 1,00 & 0,31 \\
\hline 5 & 0,50 & 0,06 & 0,63 & 0,00 & 0,50 & 0,00 & 0,94 & 0,19 & 1,00 & 0,31 & 1,00 & 0,31 \\
\hline 6 & 0,44 & 0,13 & 0,63 & 0,00 & 0,56 & 0,00 & 1,00 & 0,19 & 1,00 & 0,31 & 1,00 & 0,31 \\
\hline 7 & 0,44 & 0,00 & 0,69 & 0,00 & 0,63 & 0,00 & 1,00 & 0,25 & 1,00 & 0,19 & 1,00 & 0,38 \\
\hline 8 & 0,50 & 0,06 & 0,63 & 0,06 & 0,63 & 0,00 & 1,00 & 0,13 & 1,00 & 0,25 & 1,00 & 0,31 \\
\hline 9 & 0,63 & 0,06 & 0,50 & 0,00 & 0,56 & 0,13 & 1,00 & 0,19 & 1,00 & 0,19 & 0,94 & 0,31 \\
\hline 10 & 0,56 & 0,00 & 0,69 & 0,00 & 0,56 & 0,00 & 0,94 & 0,25 & 1,00 & 0,25 & 0,94 & 0,25 \\
\hline Média & $\mathbf{0 , 5 7}$ & 0,07 & 0,64 & $* 0,04$ & $* 0,63$ & $* 0,03$ & $* 0,98$ & 0,21 & $* 0,99$ & $* 0,25$ & $* 0,99$ & $* 0,31$ \\
\hline DP & 0,11 & 0,06 & 0,13 & 0,08 & 0,12 & 0,05 & $\mathbf{0 , 0 3}$ & 0,05 & $\mathbf{0 , 0 3}$ & 0,05 & $\mathbf{0 , 0 3}$ & 0,04 \\
\hline
\end{tabular}

Nota: Cada célula representa a média, entre os 16 sujeitos, das respostas do teste KPSS $(0 \rightarrow$ estacionário ou $1 \rightarrow$ não-estacionário), indicando o nível de estacionariedade em cada etapa e ao longo da série (neste caso, média \pm DP). ${ }^{*}$ Distribuição não-normal $(\mathrm{p}<0,05) \rightarrow$ teste de Shapiro-Wilk. Note: each cell represents the average of the KPSS test results $(0 \rightarrow$ stationary; $1 \rightarrow$ nonstationary), among the 16 subjects, indicating the level of stationarity at each stage and throughout the sets (mean $\pm S D$ ). ${ }^{*}$ Non-normal distribution $(p<0.05) \rightarrow$ Shapiro-Wilk test. 
estudo da EMG-S. Apesar da existência de certo grau de não-estacionariedade agindo sobre a faixa ILR, os valores das médias nessa faixa, segundo o teste KPSS, estão muito próximos de zero (especialmente a $60^{\circ} / \mathrm{s}$ ), o que caracteriza o comportamento de sinais "ciclo-estacionários" e, portanto, adequados às análises de EMG-S.

Ainda na Tabela 3 pode-se observar que as médias a $180^{\circ} / \mathrm{s}$, em todas as etapas e séries, são maiores que as médias a $60 \%$ s, sugerindo que quanto maior a velocidade angular, maior a não-estacionariedade do sinal de EMG-S. A Tabela 6 confirma essa hipótese

Tabela 4. Comparação estatística, entre as séries isocinéticas, dos níveis de estacionariedade nas etapas VO e ILR.

Table 4. Statistical comparison, among the isokinetic sets, of the stationarity levels in VO and ILR.

\begin{tabular}{clc}
\hline Velocidade & Etapa & Resultados \\
\hline $60 \% / \mathrm{s}$ & ${ }^{1} \mathrm{VO}$ & $\mathrm{S} 1=\mathrm{S} 2, \mathrm{~S} 2=\mathrm{S} 3, \mathrm{~S} 1=\mathrm{S} 3$ \\
& ${ }^{2} \mathrm{ILR}$ & $\mathrm{S} 1=\mathrm{S} 2, \mathrm{~S} 2=\mathrm{S} 3, \mathrm{~S} 1=\mathrm{S} 3$ \\
$180 \% \mathrm{~s}$ & ${ }^{3} \mathrm{VO}$ & $\mathrm{S} 1=\mathrm{S} 2, \mathrm{~S} 2=\mathrm{S} 3, \mathrm{~S} 1=\mathrm{S} 3$ \\
& ${ }^{4} \mathrm{ILR}$ & $\mathrm{S} 1=\mathrm{S} 2, \mathrm{~S} 2=\mathrm{S} 3, \mathrm{~S} 1<\mathrm{S} 3$ \\
\hline
\end{tabular}

Nota: nas comparações entre as séries foi utilizado o teste de Friedman com Wilcoxon Signed-Rank (post hoc). ${ }^{1}$ Aceita $\mathrm{H}_{0} \rightarrow H(3)=2,46, \mathrm{p}>0,05$, ${ }^{2}$ Aceita $\mathrm{H}_{0} \rightarrow H(3)=3,00, \mathrm{p}>0,05,{ }^{3}$ Aceita $\mathrm{H}_{0} \rightarrow H(3)=0,40, \mathrm{p}>0,05$, ${ }^{4}$ Rejeita $\mathrm{H}_{0} \rightarrow H(3)=10,75, \mathrm{p}<0,05$. Note: the Friedman test with Wilcoxon Signed Rank (post hoc) were used in the comparisons among the sets. ${ }^{1}$ Accept $H_{0} \rightarrow H(3)=2.46, p>0.05,{ }^{2}$ Accept $H_{0} \rightarrow H(3)=3.00$ $p>0.05,{ }^{3}$ Accept $H_{0} \rightarrow H(3)=0.40, p>0.05,{ }^{4}$ Reject $H_{0} \rightarrow H(3)=10.75$, $p<0.05$.

Tabela 5. Comparação estatística entre os níveis de estacionariedade das etapas VO e ILR dentro de cada série isocinética.

Table 5. Statistical comparison among the stationarity levels of VO and ILR within each isokinetic set.

\begin{tabular}{cccc}
\hline Velocidade & Série & Estacionariedade & $\begin{array}{l}\text { Média }_{\text {vo }} \text { ' } \\
\text { Média }_{\text {ILR }}\end{array}$ \\
\hline $60 \% / \mathrm{s}$ & 1 & ${ }^{1} \mathrm{VO}<\mathrm{ILR}$ & 8,27 \\
& 2 & ${ }^{2} \mathrm{VO}<\mathrm{ILR}$ & 17,00 \\
& 3 & ${ }^{2} \mathrm{VO}<\mathrm{ILR}$ & 20,00 \\
$180 \% \mathrm{~s}$ & 1 & ${ }^{2} \mathrm{VO}<\mathrm{ILR}$ & 4,62 \\
& 2 & ${ }^{2} \mathrm{VO}<\mathrm{ILR}$ & 3,95 \\
& 3 & ${ }^{2} \mathrm{VO}<\mathrm{ILR}$ & 3,16
\end{tabular}

Nota: comparações com diferenças significativas $(\mathrm{p}<0,05):{ }^{1} t$-Student $\mathrm{e}^{2}$ Wilcoxon-Signed-Rank. Nestes casos, média maior significa menor nível de estacionariedade. Note: comparisons with significant differences $(p<0.05)$ : ${ }^{1} t$-Student and ${ }^{2}$ Wilcoxon-Signed-Rank. The higher the average, the lower the stationarity level. e mostra que os efeitos não-estacionários podem ser até $10(1,73)$ vezes maiores para ILR (VO) quando a velocidade de $180 \%$ s é comparada à de $60 \%$ s. Isso pode ser explicado pelo fato de que a inércia de um movimento a $180 \%$ sé maior que a $60 \%$, exigindo do dinamômetro isocinético um esforço também maior para ajustar a velocidade angular a um patamar constante e pré-definido, o que aumenta a probabilidade de ocorrência de artefatos de movimento (não-estacionariedade rápida).

Quando a mesma etapa isocinética foi comparada entre as séries de uma mesma velocidade angular, conforme ilustrado pela Tabela 4 , nenhuma diferença significativa foi encontrada, exceto entre as séries 1 e 3 para ILR a $180^{\circ} / \mathrm{s}(\mathrm{S} 1<\mathrm{S} 3)$. Isso se deve ao fato de que a série 3 apresenta níveis maiores de nãoestacionariedade em função de estado de fadiga mais acentuado, o que não ocorre nas séries 1 e 2 . $\mathrm{Na}$ velocidade de $60^{\circ} \mathrm{s}$, a manifestação da fadiga já acontece com intensidade relativa desde a série 1 , razão pela qual não são evidenciadas diferenças significativas entre as séries. Os resultados encontrados sugerem que, nas condições de contração dinâmica propostas neste estudo, os efeitos perturbadores do sinal de EMG-S são cíclicos e praticamente invariantes, ocorrendo com maior intensidade na etapa VO. Por consequência, os sinais de EMG-S podem ser satisfatoriamente considerados ciclo-estacionários na etapa ILR, conforme preliminarmente observado por Schwartz e Nascimento (2011).

\section{Conclusão}

Uma vez constatados elevados níveis de nãoestacionariedade na etapa $\mathrm{VO}$, em contrapartida a relevantes níveis de estacionariedade na etapa ILR, fica fortalecida a recomendação de que a análise de EMG-S associada a testes isocinéticos de extensão do joelho deva ocorrer somente na faixa correspondente a ILR, descartando-se a informação contida em VO. Isso é coerente com o estudo de Schwartz et al. (2010) que sugere que a interpretação dos dados de testes isocinéticos seja restrita à faixa de velocidade constante (ILR), o que condiz com a própria definição

Tabela 6. Comparação do nível de estacionariedade entre as velocidades $60^{\circ} / \mathrm{s}$ e $180^{\circ}$ s considerando as etapas VO e ILR dentro de cada série isocinética.

Table 6. Comparison of the stationarity level between speeds $60 \%$ and $180 \%$ considering VO and ILR phases within the isokinetic set.

\begin{tabular}{cll}
\hline Série & VO & \multicolumn{1}{c}{ ILR } \\
\hline 1 & $\mathrm{VO}_{60}<\mathrm{VO}_{180},\left(\mathrm{VO}_{180} / \mathrm{VO}_{60}\right)=1,73$ & $\mathrm{ILR}_{60}<\mathrm{ILR}_{180},\left(\mathrm{ILR}_{180} / \mathrm{ILR}_{60}\right)=3,09$ \\
2 & $\mathrm{VO}_{60}<\mathrm{VO}_{180},\left(\mathrm{VO}_{180} / \mathrm{VO}_{60}\right)=1,55$ & $\mathrm{ILR}_{60}<\mathrm{ILR}_{180},\left(\mathrm{ILR}_{180} / \mathrm{ILR}_{60}\right)=6,67$ \\
3 & $\mathrm{VO}_{60}<\mathrm{VO}_{180},\left(\mathrm{VO}_{180} / \mathrm{VO}_{60}\right)=1,58$ & $\mathrm{ILR}_{60}<\mathrm{ILR}_{180},\left(\mathrm{ILR}_{180} / \mathrm{ILR}_{60}\right)=10,00$ \\
\hline
\end{tabular}

Nota: todas as comparações apresentaram diferenças significativas $(\mathrm{p}<0,05)$ com o teste Wilcoxon-Signed-Rank. A não-estacionariedade é maior a $180 \%$ s para ambas as etapas. Note: all comparisons showed significant differences $(p<0.05)$ by Wilcoxon-Signed-Rank test. Greater non-stationarity was found at $180 \%$ in both steps. 
do termo isocinético. Estudos futuros poderiam avaliar os efeitos do artefato de oscilação da velocidade em outras articulações e velocidades angulares.

\section{Referências}

Basmajian JV, De Luca CJ. Muscle alive: their function revealed by electromyography. 5th ed. Baltimore: Williams \& Wilkins; 1985.

Bilodeau M, Arsenault AB, Gravel D, Bourbonnais D. The influence of an increase in the level of force on the EMG power spectrum of elbow extensors. European Journal of Applied Physiology. 1990; 61(5-6):461-6. PMid:2079067. http://dx.doi.org/10.1007/BF00236068

Bonato P, Roy SH, Knaflitz M, De Luca CJ. Time-frequency parameters of the surface myoelectric signal for assessing muscle fatigue during cyclic dynamic contractions. IEEE Transactions on Biomedical Engineering. 2001; 48(7):745-53. PMid:11442286. http://dx.doi.org/10.1109/10.930899

Broman H, Bilotto G, De Luca CJ. Myoelectric signal conduction velocity and spectral parameters: Influence of force and time. Journal of Applied Physiology. 1985; 58(5):1428-37. PMid:3997710.

Brown LE, editor. Isokinetics in human performance. Champaign: Human Kinetics; 2000.

Brown LE, Whitehurst M, Findley BF, Gilbert R, Buchalter $\mathrm{DN}$. Isokinetic load range during shoulder rotation exercise in elite male junior tennis Players. Journal of Strength and Conditioning Research. 1995a; 9(3):139-43.

Brown LE, Whitehurst M, Gilbert R, Buchalter DN. The effect of velocity and gender on load range during knee extension and flexion exercise on an isokinetic device. Journal of Orthopaedic and Sports Physical Therapy. 1995b; 21(2):107-12.

De Luca CJ. The use of surface electromyography in biomechanics. Journal of Applied Biomechanics. 1997; 13(2):135-63.

De Sá JPM. Applied statistics using SPSS, STATISTICA, MATLAB and R. 2th ed. Germany: Springer-Verlag; 2007.

Farina D, Pozzo M, Merlo E, Bottin A, Merletti R. Assessment of average muscle fiber conduction velocity from surface EMG signals during fatiguing dynamic contractions. IEEE Transactions on Biomedical Engineering. 2004; 51(8):1383-93. PMid:15311823. http://dx.doi.org/10.1109/TBME.2004.827556

Inbar GF, Allin J, Kranz H. Surface EMG spectral changes with muscle length. Medical and Biological Engineering and Computing. 1987; 25(6):683-9. http://dx.doi.org/10.1007/ BF02447340

Komo JJ. Random signal analysis in engineering systems. Orlando: Academic Press, Inc.; 1987.
Kwiatkowiski D, Phillips PCB, Schmidt P, Shin Y. Testing the null hypothesis of stationarity against the alternative of a unit root. How sure are we that economic time series have a unit root? Journal of Econometrics. 1992; 54:158-78.

Maganioti AE, Chrissanthi HD, Charalabos PC, Andreas RD, George PN, Christos CN. Cointegration of eventrelated potential (ERP) signals in experiments with different electromagnetic field (EMF) conditions. Health. 2010; 2(5):400-6. http://dx.doi.org/10.4236/health.2010.25060

Masuda T, Miyano H, Sadoyama T. The position of innervation zones in the biceps brachii investigated by surface electromyography. IEEE Transactions on Biomedical Engineering. 1985; BME32(1):36-42. PMid:3980027. http:// dx.doi.org/10.1109/TBME.1985.325614

McNair PJ, Depledge J, Brettkelly M, Stanley SN. Verbal encouragement: effects on maximum effort voluntary muscle action. British Journal of Sports Medicine. 1996; 30(3):243-5. http://dx.doi.org/10.1136/bjsm.30.3.243

Papoulis A, Pillai SU. Probability, random variables and stochastic processes. 4th ed. New York: McGraw-Hill Higher Education; 2002.

Sapega AA, Nicholas JA, Sokolow D, Saranti A. The nature of torque overshoot in Cybex isokinetic dynamometry. Medicine and Science in Sports and Exercise. 1982; 14(5):368-75. http:// dx.doi.org/10.1249/00005768-198205000-00010

Schwartz FP, Bottaro M, Celes RS, Brown LE, Nascimento FAO. The influence of velocity overshoot movement artifact on isokinetic knee extension tests. Journal of Sports Science and Medicine. 2010; 9(1)140-6.

Schwartz FP, Nascimento FAO, Bottaro M, Celes RS. Arquitetura para o processamento integrado de sinais biomecânicos e eletromiográficos. Revista Brasileira de Engenharia Biomédica. 2011; 27(1):24-38. http://dx.doi. org/10.4322/rbeb.2011.003

Schwartz FP, Nascimento FAO. Stationarity of Surface electromyographic signals on isokinetic knee extension test. In: Health Care Exchanges (PAHCE), 2011 Pan American: Proceedings of the VI Pan American Health Care Exchange; 2011 Mar 28 - Apr 1; Rio de Janeiro, Brasil. p. 49-9. doi: http://dx.doi.org/10.1109/PAHCE.2011.5871845

Schwartz FP, Rocha Junior VA, Rocha AF, Nascimento FAO. Ferramenta computacional para o processamento de sinais eletromiográficos e variáveis isocinéticas. In: Barbosa EM, Souza MCD, Souza ER, editors. CBEB 2008: Proceedings of the XXI Congresso Brasileiro de Engenharia Biomédica; 2008 Nov 16-20; Salvador, Brasil. São Carlos: Cubo Multimídia; 2008. p. 1699-702.

Smith SW. The scientist and engineer's guide to digital signal processing. [Internet]. 1997. [cited 2011 Out 02]. Available from:www.dspguide.com/ch3/2.htm

Zivot E, Wang J. Modeling financial time series with S-Plus. New York: Springer; 2002.

\section{Autores}

Fabiano Peruzzo Schwartz, Francisco Assis de Oliveira Nascimento

Departamento de Engenharia Elétrica, Universidade de Brasília - UnB, Brasília, DF, Brasil

Martim Bottaro, Rodrigo Souza Celes

Faculdade de Educação Física, Universidade de Brasília - UnB, Brasília, DF, Brasil 